\title{
Realizing the benefits of restored periodicity in the advanced light source
}

\author{
D. Robin \\ Lawrence Berkeley National Laboratory, Berkeley, California 94720 \\ J. Safranek \\ Stanford Linear Accelerator Center, Stanford, California 94309 \\ W. Decking \\ Lawrence Berkeley National Laboratory, Berkeley, California 94720
}

(Received 31 August 1998; published 21 April 1999)

\begin{abstract}
An essential feature of third generation storage ring based light sources is the magnetic lattice is designed with a high degree of periodicity. Tracking simulations show that if the periodicity is perturbed (by focusing errors, for example), nonlinear resonances become excited, which causes a reduction in the dynamic aperture. Therefore it is important to have a method to measure and correct perturbed periodicity. In this paper we study the effect of broken and restored periodicity at an actual third generation light source: the Advanced Light Source at Lawrence Berkeley National Laboratory. First, we show that it is possible to accurately determine the storage ring optic and thus the perturbation of the periodicity by fitting measured orbit response matrices. This method allows us to determine individual field gradient errors in quadrupoles and closed orbit errors in sextupoles. By varying individual quadrupole field strengths it is possible to correct the optic, largely restoring the lattice periodicity. A comparison is made of the performance of the storage ring before and after the optic is corrected. Measurements of the electron beam tails and the synchrotron light image reveal a large suppression in resonance excitation after the optic is corrected. Correcting the optic also improves the injection efficiency. [S1098-4402(99)00037-3]
\end{abstract}

PACS numbers: 29.27.Bd, 29.27.Fh, 29.20.Dh

\section{INTRODUCTION}

The Advanced Light Source (ALS) is a 1.0 to $2.0 \mathrm{GeV}$ storage ring based synchrotron light source located at Lawrence Berkeley National Laboratory [1]. Similar to other third generation light sources, the ALS storage ring was designed to produce small beam emittances to increase the brightness of radiation emitted from insertion devices. The small beam emittances are created by strongly focusing the beam with high field quadrupole magnets. These quadrupole magnets generate large chromatic aberrations that need to be corrected with high field sextupole magnets. These sextupole magnets in turn introduce geometrical and higher order chromatic aberrations that can result in undesirable dynamic behavior of the electrons - such as excitation of structural resonances and chaotic motion. This nonlinear behavior can lead to: 1. small dynamic aperture (a) short lifetimes (Touschek and gas-scattering) and (b) slow injection rates; 2. distortion in the beam shape; and 3. reduction in the betatron tune space where beam can stably circulate.

There is an important feature of the ring design that helps suppress resonance excitation - the ring is designed with a high degree of periodicity. The ALS ring is 12 -fold periodic. Figure 1 shows one twelfth of the ring consisting of 3 dipole magnets $(B) 6$ quadrupoles $(Q F, Q D$, and $Q F A)$, and 4 sextupole magnets $(S F$ and $S D$ ). The locations of horizontal and vertical steering magnets, and beam posi- tion monitors (BPMs) are also shown. The dipole magnets have quadrupole fields in addition to their dipole fields (i.e., combined function magnets). The magnetic lattice is a triple bend achromat that is mirror symmetric about its center.

Ideally, each of the 12 sectors is identical. In such a ring, structural betatron resonances may occur when the following condition is satisfied:

$$
N_{x} \frac{\nu_{x}}{12}+N_{y} \frac{\nu_{y}}{12}=M,
$$

where $\nu_{x}$ and $\nu_{y}$ are the horizontal and vertical betatron tunes for the full ring and $N_{x}, N_{y}$, and $M$ are integers. In a ring with no periodicity the full ring is the basic unit of the machine. In that case structural betatron resonances may occur when

$$
N_{x} \nu_{x}+N_{y} \nu_{y}=M,
$$

which is much less restrictive than the condition given by Eq. (1).

In this paper, we define the resonance conditions given in Eq. (1) as resonances that are "allowed" by periodicity. All conditions given in Eq. (2), excluding those that are given in Eq. (1), we define as resonances that are "unallowed" by periodicity. To illustrate the reduction in the number of low-order resonances with 12fold periodicity, all resonances up to fifth order $\left(\left|N_{x}\right|+\right.$ $\left.\left|N_{y}\right| \leq 5\right)$ are plotted in the left side of Fig. 2 and the 


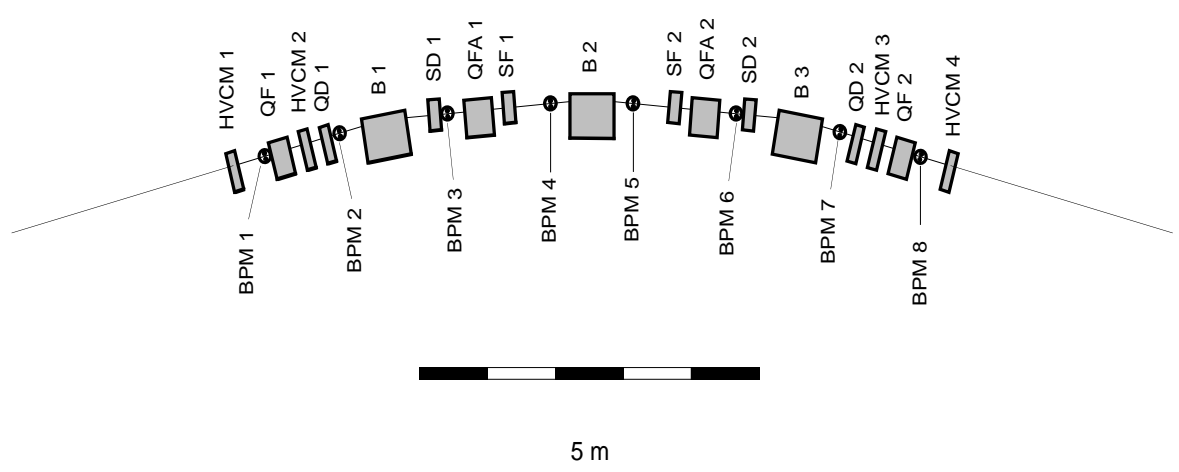

FIG. 1. Typical ALS storage ring sector.

allowed resonances up to fifth order are plotted in the right side of Fig. 2.

Unallowed resonances are excited when focusing errors, such as quadrupole field errors and orbit errors in sextupoles, perturb the periodicity of the ring. The strength of resonance excitation depends upon the degree of periodicity breaking. The periodicity is broken to some extent in every storage ring. In practice we would like to know: 1. Can we identify the cause of the periodicity breaking? 2. How bad is the periodicity broken? 3. Can we restore the periodicity? 4. How is the beam dynamics affected by the broken periodicity? 5 . How is the beam dynamics improved by the restored periodicity?

Through experimental studies we were able to answer these questions. In this paper we present the results of the studies. In Sec. II we describe the technique of modeling measured orbit response matrix data that was used to determine the cause of periodicity breaking. We present the results of our modeling to find individual quadrupole gradient errors (Sec. III) and horizontal orbit offsets in sextupoles (Sec. IV). In Sec. V we describe how the linear optic was corrected and the ring's periodicity was restored by adjusting individual quadrupole gradients in the ring.
In Secs. VI and VII we compare the performance of the storage ring before and after the optic was corrected. In particular, we measure improved injection efficiency (Sec. VI) and a reduction in resonance excitation (Sec. VII). In Sec. VIII we end with some concluding remarks.

\section{FITTING THE MODEL}

The magnet gradient distribution in the ALS was determined by analyzing measured orbit response matrix data. The analysis method is one suggested by Corbett, Lee, and Ziemann at Stanford Linear Accelerator Center [2] and refined by Safranek at Brookhaven National Laboratory $[3,4]$. The first analysis of ALS orbit response matrices was made in 1994 shortly after commissioning by Bengtsson and Meddahi [5] and later by Robin et al. [6].

An orbit response matrix, $\mathbf{M}$, is defined by

$$
\left(\begin{array}{l}
\vec{x} \\
\vec{y}
\end{array}\right)=\mathbf{M}\left(\begin{array}{c}
\vec{\theta}_{x} \\
\vec{\theta}_{y}
\end{array}\right)
$$

where $\vec{\theta}_{x}, \vec{\theta}_{y}$ are changes in steering magnet strengths and $\vec{x}, \vec{y}$ are the resulting electron orbit perturbations at the beam position monitors (BPMs). The measured
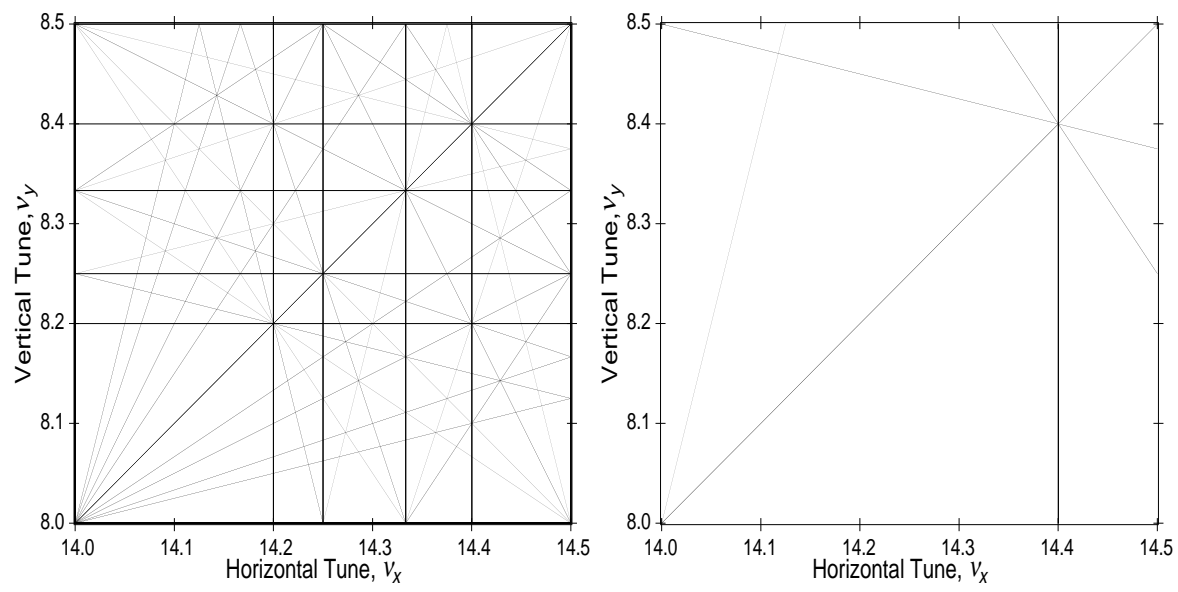

FIG. 2. Resonance diagram: All betatron resonances up to 5th order (left); all "allowed" resonances up to 5th order with 12-fold periodicity (right). 
orbit response matrix, $\mathbf{M}_{\text {meas }}$, is obtained by changing a steering magnet and measuring orbit changes with BPMs. Also, it is possible to calculate the orbit response matrix, $\mathbf{M}_{\text {mod }}$, assuming some gradient distribution in the storage ring and using an optic model. If the assumed gradient distribution is wrong, then $\mathbf{M}_{\text {meas }}$ and $\mathbf{M}_{\text {mod }}$ will not be the same. The actual gradient distribution in the ring is then determined by adjusting the gradient distribution in the model to minimize the difference between $\mathbf{M}_{\text {meas }}$ and $\mathbf{M}_{\text {mod }}$. To calculate $\mathbf{M}_{\text {mod }}$ we used the optic code COMFORT [7].

The parameters in the COMFORT model were varied to minimize the $\chi^{2}$ deviation between the model and measured orbit response matrices using the computer code LOCO [4]:

$$
\chi^{2}=\sum_{i, j} \frac{\left(\mathrm{M}_{\text {mod }, i j}-M_{\text {meas }, i j}\right)}{\sigma_{\mathrm{i}}^{2}},
$$

where the summation is over the BPMs, $i$, and the steerers, $j$. The $\sigma_{i}$ are the measured rms noise levels for the BPMs. In the ALS there are 94 horizontal correctors, 70 vertical correctors, 96 horizontal BPMs, and 96 vertical BPMs. This means that there are $(96+$ 96) $\times(94+70)=31488$ elements in the fully coupled matrix or $(94 \times 96)+(70 \times 96)=15744$ elements in the uncoupled matrix. In this work we did not attempt to determine the skew gradient components in the ring. Therefore we ignore the cross terms in the matrix and fit only the uncoupled matrix elements.

If the beam orbit is not steered horizontally through the center of the sextupoles there is a quadrupole feeddown component of the sextupole field proportional to both the horizontal offset and the integrated strength of the sextupole. At the ALS it is possible to store beam and measure an orbit response matrix with the sextupole magnets turned off. This is convenient because it allows us to fit that matrix without varying the sextupoles' quadrupole gradients. In that way, we are able to find the quadrupole gradients independently of the sextupoles. Then we measure an orbit response matrix with the sextupoles turned on and fit the data by varying the sextupole gradients with the quadrupole gradients kept fixed in the model. In this way we are able to minimize the number of parameters used to fit each response matrix. So in gathering the data we make a back-toback measurement of two response matrices: one with the sextupoles turned off and another with the sextupoles turned on.

First, we discuss the gradient parameters used to fit the data set with the sextupole magnets turned off. There are 108 magnets in the storage ring that have quadrupole components: $36 \mathrm{Bs}, 24 Q F \mathrm{~s}, 24 Q D \mathrm{~s}$, and $24 Q F A \mathrm{~s}$ (see Fig. 1). These magnets are powered by 50 power supplies: One supply powers all the $B \mathrm{~s}$, one supply powers all $Q F A$ s, and the remaining 48 supplies individually power the $24 Q F$ and $24 Q D$ magnets. In our fit we varied only
50 quadrupole field strengths, one associated with each of the quadrupole supplies. We did not choose to vary all 108 parameters for two reasons. First, the noise of the data is not sufficiently small to accurately distinguish gradient errors in all individual magnets. Second, we assume that gross quadrupole errors would most probably be due to differences in power supplies and not to mechanical differences in the magnets [8].

The parameters used to fit the data set with the sextupoles turned off are the quadrupole field strengths (50), the BPM gains (192), and the steerer magnets gains (164). In addition, there is another set of parameters used in the fit: the energy shifts associated with changes in steerer magnets. This energy shift occurs when the field of a horizontal steerer magnet located in a dispersion region is changed [9]

$$
\frac{\Delta E}{E}=-\frac{\theta_{x} \eta_{x}}{\alpha L_{0}},
$$

where $\theta_{x}$ is the change in steerer magnet strength, $\eta_{x}$ is the dispersion at the location of the steerer magnet, $\alpha$ is the momentum compaction, and $L_{0}$ is the circumference of the ring. This adds another 94 parameters to the fit. A total of 500 parameters are varied to minimize $\chi^{2}$.

After we fit the response matrix data, we fit the response matrix data set taken with the sextupoles turned on. The chromaticity is adjusted using two families of sextupoles, $S F$ and $S D$. To fit this data we fix the values for the quadrupoles that we calculated from the previous set and only vary quadrupole gradients associated with each of the $24 S F$ and $24 S D$ sextupole magnets. In addition to the 48 quadrupole gradients we vary the 192 BPM gains, 164 corrector gains, and the 94 energy shifts. So in the second iteration there are 498 parameters that are varied to minimize $\chi^{2}$. For more details concerning the LOCO fit we refer the reader to another paper [4].

\section{QUADRUPOLE FIELD ERRORS}

Initial LOCO analyses of the ALS storage ring were made in the fall of 1995. To generate the measured response matrix the magnitude of the steerer magnet changes were chosen to produce approximately a $0.8 \mathrm{~mm}$ rms change horizontally and a $1 \mathrm{~mm}$ rms change vertically in the orbit at the BPMs. The initial LOCO analysis was made for quadrupoles without the sextupole magnets turned on. Before the fit the disagreement between the measured and modeled orbit changes is greater than $100 \mu \mathrm{m}$ rms horizontally and vertically. After fitting the model the agreement between the measured and modeled orbit changes is $13 \mu \mathrm{m} \mathrm{rms} \mathrm{horizontally} \mathrm{and} \mathrm{vertically.} \mathrm{This} \mathrm{should}$ be compared with the measured rms noise of the BPMs, $\sigma_{i}$, which was $12 \mu \mathrm{m}$ horizontally and $10 \mu \mathrm{m}$ vertically. Therefore the fits nearly converged down to the noise. An example of the agreement between the model and machine for a typical orbit change before and after the fit can be seen in Fig. 3. 


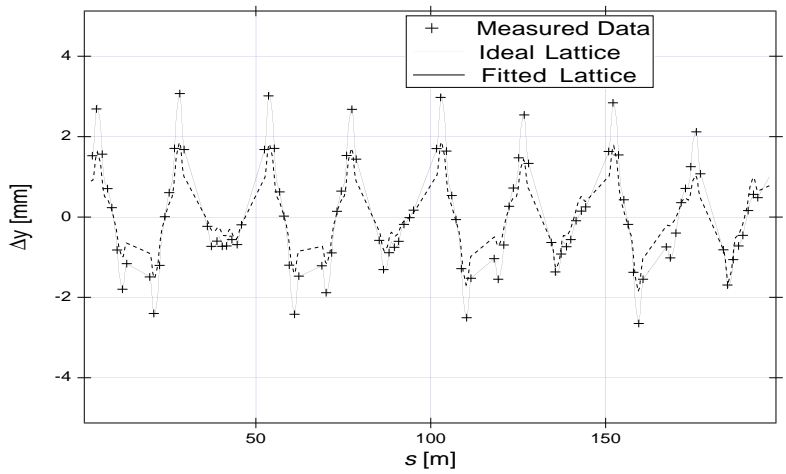

FIG. 3. A comparison of 1 column of the response matrix (+) with the ideal lattice and with the lattice after fitting.

Although the accuracy of the method is difficult to determine, there was only a $0.1 \%$ rms variation in the fit quadrupole gradients from data set to data set. The fits revealed that the $Q D$ family had significantly larger variation in field than the $Q F$ family. In particular, the rms variation in quadrupole field strengths within each quadrupole family was determined to be $0.20 \%$ for the $Q F$ quadrupoles and $0.63 \%$ for the $Q D$ quadrupoles. These $Q D$ values are particularly large when compared with the quadrupole design tolerances which specify that within a family the field variation should lie within a band of $\pm 0.2 \%$ [10].

As mentioned in the previous section, we assumed that any large variation in quadrupole fields is more likely a result of the variation in the power supply than in the mechanical construction. Independently, each magnet power supply excitation current was measured using a handheld current monitor [11]. A magnet-to-magnet comparison was made between the quadrupole strengths obtained with the current meter and the response matrix fitting. The results are shown in Fig. 4. The agreement is within the precision of the current monitor $( \pm 0.3 \%)$. In fact, the rms difference between the two measurements is $0.2 \%$. Since these two independent measurement methods produced consistent results, it gave us confidence that the two methods are capable of pinpointing individual magnet field strength errors to a precision better than $0.3 \%$. In particular, both measurements showed that 4 of the $24 Q D$ magnets were more than $1 \%$ lower than the average (magnets 9, 13, 16, and 22 in Fig. 4). It was ultimately shown that the reason that power supplies were not regulating to specification was due to malfunctioning regulating shunts.

Without sextupoles we identified the major cause of periodicity breaking, thus answering the first question posed in the introduction. The second question posed was how bad the periodicity was broken. One measure of the periodicity breaking is the distortion of the $\beta$ function in the ring. Using the calibrated model it is possible to compute the $\beta$ function. The rms perturbation of the $\beta$ function from the ideal $\beta$ function is $3 \%$ horizontally and $15 \%$ vertically. The perturbation of the $\beta$ function is displayed in Fig. 5(top).

In 1996 we improved the quality of our measured response matrices. Prior to 1996 only one measurement of the orbit was taken after each steering magnet was changed. To reduce the noise level of the BPMs, 500 measurements of the orbit were taken after each steering magnet was changed. These 500 orbits were then averaged. As expected the noise level was reduced by the square root of the number of averages from greater than $10 \mu \mathrm{m}$ to less than $1 \mu \mathrm{m}$. At the time of this paper the agreement between the measured and the modeled response matrices is $4 \mu \mathrm{m}$ horizontally and vertically. The fits still do not converge down to the noise level. In a system as complex as a particle accelerator it is practically impossible to eliminate all systematic errors. In particular, we did not account for energy dependent $\beta$ functions; roll angle in BPMs, correctors, and quadrupoles; nonlinearities in BPM gains, etc.

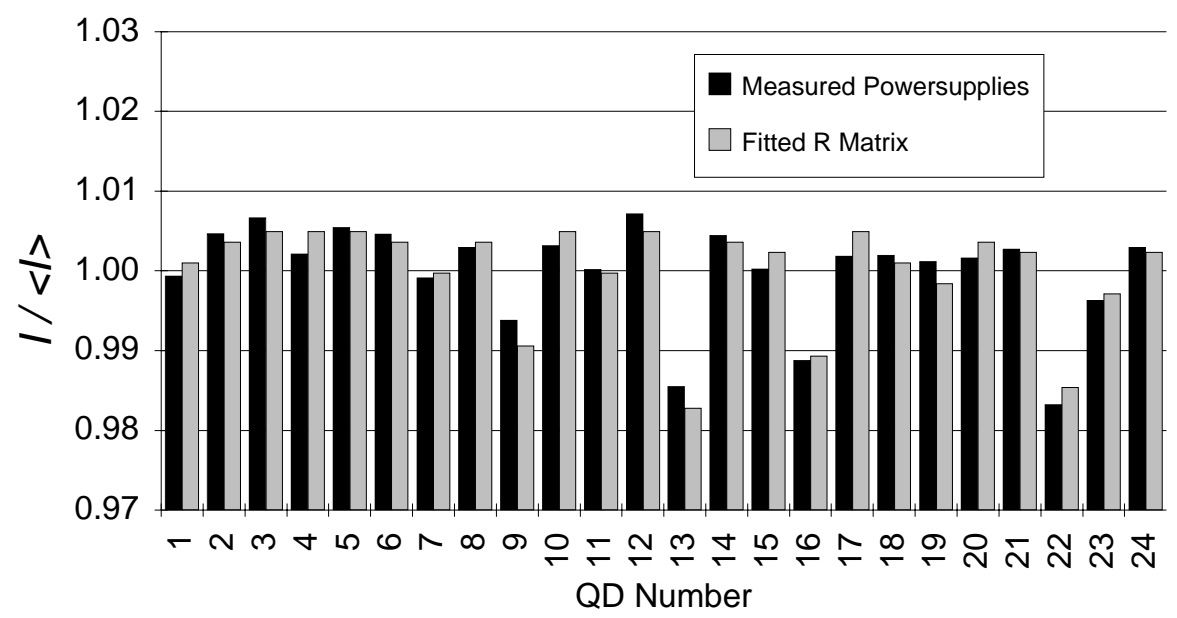

FIG. 4. A comparison of the relative variation of $Q D$ quadrupole strengths as measured with a current monitor and fitted response matrix. 

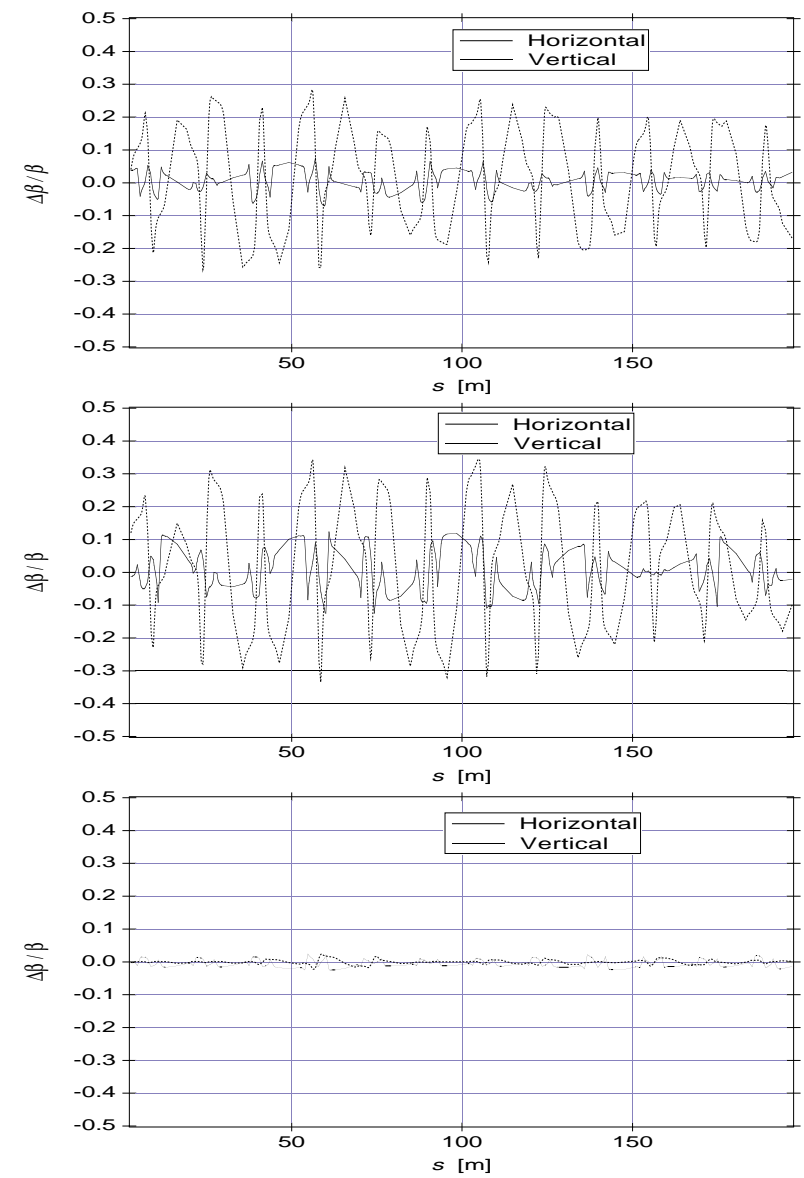

FIG. 5. Horizontal and vertical $\beta$ beating with sextupoles turned off (top), with sextupoles turned on (middle), and with sextupoles turned on after optic is corrected (bottom).

\section{ORBIT ERRORS IN SEXTUPOLES}

As was mentioned previously, if the orbit of the beam in the sextupoles is horizontally displaced from the magnetic center, then there is a quadrupole component of the field. The vector potential (normalized by the magnetic rigidity), $A_{z}$, of a sextupole is

$$
A_{z}=-\frac{S}{3}\left(x^{3}-3 x y^{2}\right),
$$

where $S$ is the integrated sextupole strength in units of $\mathrm{m}^{-2}$ and $x$ and $y$ are measured with respect to the magnetic center of the sextupoles. If there is a horizontal closed orbit distortion, $x_{0}$, in the sextupoles, then the equivalent quadrupole component, $K_{s}$ in a sextupole is

$$
K_{s}=2 S x_{0} \text {. }
$$

Given the sextupole's integrated strength, $S$, and integrated gradient, $K_{S}$, measured with respect to the beam's closed orbit, one knows $x_{0}$, the position of the beam with respect to the magnetic center of the sextupole.

To determine $x_{0}$, first the response matrix with sextupoles turned on is fitted, keeping the quadrupole gradients fixed to the value determined previously with sextupoles turned off. From this fit we determine the $48 K_{s}$. Second, the sextupole gradients, $S$, are determined by adjusting the sextupoles to correct the linear chromaticity to zero in the model. One power supply powers all of the $S F$ sextupoles and one power supply powers all of the $S D$ sextupoles. In our fits we assumed one sextupole strength for all the $24 S F$ sextupoles and one sextupole strength for all the $24 S D$ sextupoles. Based upon similar assumptions made about the quadrupoles in the previous section, the assumption is made that there is little variation in $S$ among the sextupoles in a family. Having values of $K_{s}$ and $S$ for each of the sextupoles, Eq. (7) is used to determine the offsets, $x_{0}$.

The offsets in the sextupoles, which were as much as $1.5 \mathrm{~mm}$ in the $S F \mathrm{~s}$ and $0.8 \mathrm{~mm}$ in the $S D \mathrm{~s}$, resulted in an additional increase in the amplitude of beating of the $\beta$ function. As can be seen in Fig. 5(middle) the rms perturbation of the $\beta$ function is $6 \%$ horizontally and $19 \%$ vertically. Even though there is some increase in the distortion of the $\beta$ function, most of the distortion was a result of the mispowered quadrupoles.

How do we trust that we have found the actual offsets of the beam with respect to the magnetic center? We tested the fits by moving the orbit in the sextupoles by a known amount and checked to see if the change in the fitted offset before and after the orbit is moved correctly reflects the change in orbit. This is done by measuring and fitting three separate response matrices. First, we measure an orbit response matrix with the sextupoles turned off and fit the quadrupole gradients in all the quadrupoles. Then we switch on the sextupoles. The sextupoles are powered so that the measured chromaticity is zero in both planes. Then we measure a second response matrix and fit the quadrupole gradients of the sextupoles. Next, we distort the horizontal closed orbit by changing one horizontal corrector to give roughly a $1 \mathrm{~mm}$ change in the orbit peakto-peak. To do this we changed the correctors excitation current by $3 \mathrm{~A}$. A new response matrix is measured and the quadrupole gradients of the sextupoles are refitted.

After the response matrices are fit, the horizontal beam offset in the sextupoles is computed for each sextupole before and after the horizontal orbit is changed. The change in the orbit is then just the difference between the horizontal offsets before and after the corrector was changed.

By modeling the orbit response matrices, we can determine the change in orbit in a second way. From the fitted model we determine that a $3 \mathrm{~A}$ change in the corrector corresponded to a 0.2 mrad kick. Using the fitted model we can predict how much a 0.2 mrad corrector kick will change the closed orbit at the sextupoles.

So we determine the orbit change at the sextupoles in two ways: one by fitting the quadrupole gradient in the sextupoles, and the other by fitting the gains of the correctors. A comparison of the two methods is shown in Fig. 6. For a $0.56 \mathrm{~mm} \mathrm{rms} \mathrm{shift} \mathrm{in} \mathrm{orbit,} \mathrm{the} \mathrm{orbit} \mathrm{changes}$ 


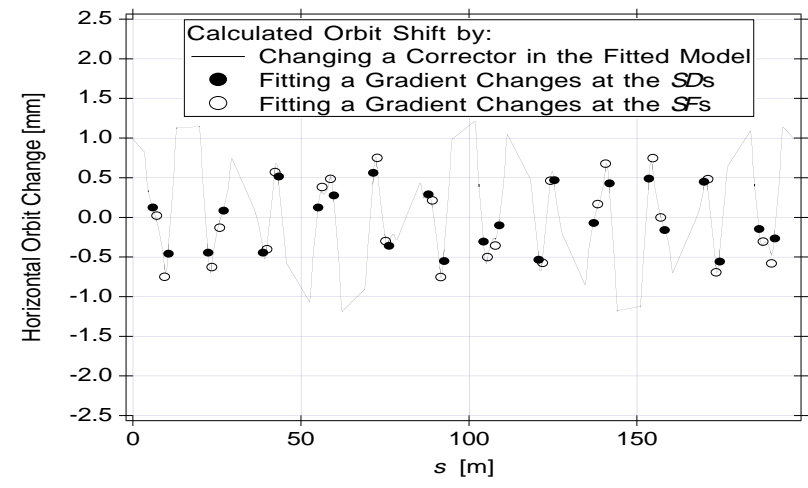

FIG. 6. Comparison of two methods for determining an orbit change in the ALS when one corrector is changed. Method 1 (solid line): Use the fitted model of the ring and the fitted gain of the corrector to calculate the orbit shift. Method 2 (circles): Use the calculated orbit change from the difference in the quadrupole gradients in the sextupoles.

at the sextupoles agree to within $75 \mu \mathrm{m}$. This gives us confidence that the absolute offset is found to reasonable accuracy $(\sim 100 \mu \mathrm{m})$.

There are two assumptions in the fit. One is that each member of the sextupole family has the same integrated strength. As discussed earlier, this is a reasonable assumption. The other assumption is that the gain of the corrector is determined correctly. As discussed in Ref. [4], the absolute gains of the correctors and the BPMs could both be off by a constant factor that cannot be determined in the fit. The good agreement between the two methods suggests that the assumptions made about the magnet-to-magnet variation of the sextupoles in a family and the absolute corrector gain are good.

\section{RESTORING THE PERIODICITY OF THE LATTICE}

Once the real lattice is known it is then possible to adjust the quadrupoles to correct the optic and restore the lattice periodicity. Choosing how the quadrupoles get set depends on what is important. For instance, it may be important to restore the $\beta$ function to restore the beam size back to ideal. Or it may be important to have the linear transfer functions between the sextupoles the same to restore the dynamic aperture [12]. Doing this may mean that the $\beta$ function may vary considerably from ideal. This happens in the situation that there is one large gradient error in the ring and it cannot be corrected locally. In our case we chose the following merit function: Adjust the quadrupoles to minimize the difference between the actual and ideal response matrix. Because we have distributed gradient errors this merit function effectively corrects both the $\beta$ beating and the transfer matrices between sextupoles.

Determining the quadrupole values that best correct the optic is also done with LOCO, and the algorithm is as follows. We have 49 individual parameters that can be adjusted to compensate the $\beta$ beating in the ring: each one of the $Q F$ and $Q D$ quadrupole magnets which can be adjusted individually and the $Q F A$ quadrupoles that can be adjusted as a group. We fit the orbit response matrix with sextupoles on keeping the gradients in the sextupoles zero in the fit model and varying only the 49 quadrupole gradient parameters. We then find the variation in those 49 parameters that best reproduces the break in periodicity of the measured response matrix. We then adjust the quadrupole power supplies in the ring with just the opposite variation. A new set of response matrices is measured then and the model is refit. From this we compute the $\beta$ beating of the lattice and find that it has reduced to less than $1 \%$ rms [see Fig. 5(bottom)]. Thus we have answered question 3 posed in the introduction. Now we move on to answer questions 4 and 5: What is the effect on the beam dynamics from the broken periodicity, and how does the behavior change when the optic is corrected?

\section{EFFECT OF PERIODICITY BREAKING ON INJECTION EFFICIENCY}

Experimentally, we found that correcting the optic dramatically improved our injection rates. To explain the experiment, we first need to describe the injection process. An illustration of the injection process is given in Fig. 7. Plot (a) shows the situation before injection. The stored beam is centered in the ring with a magnetic septum located $21 \mathrm{~mm}$ horizontally inward (to the right). Plot (b) shows the situation during injection. The stored beam is bumped $15 \mathrm{~mm}$ inward towards the septum. At the same time the injected beam enters the ring on the opposite side of the septum. The duration of the bump lasts about $4 \mu \mathrm{s}$ (a few turns) after which both the injected and the stored beam are clear (to the left) of the septum.

The storage ring acceptance drawn in Figs. 7(a) and 7(b) is $15 \mathrm{~mm}$. As plot (b) indicates, when the stored beam is locally bumped, the injected beam just falls inside the acceptance. As the bump or the aperture becomes smaller fewer injected particles are captured. Conversely, the amplitude of the bump cannot be too large or the stored beam will strike the septum. This will cause some or all of the stored beam to be lost.

Before the optic of the lattice was corrected, we were experiencing a low injection efficiency. In order to get a reasonable injection rate we needed to create a distortion of the closed orbit of about $4 \mathrm{~mm}$ towards the septum [see Figs. 7(c) and 7(d)]. This indicated a smaller than expected acceptance. Putting in a $4 \mathrm{~mm}$ distortion in the orbit gave us the maximum injection efficiency. If we increased the amplitude of the bump the injection rate went down, presumably because we were scraping some of the stored beam off the septum. We had a 
(a)

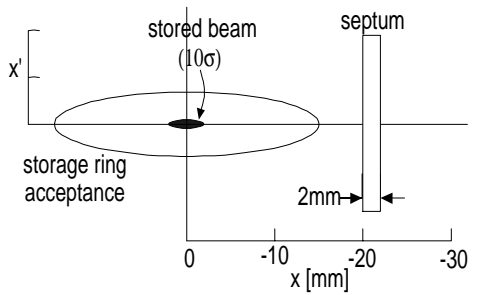

(c)

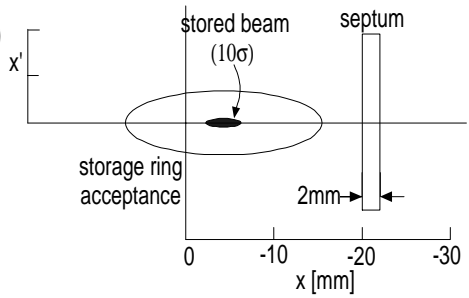

(b)

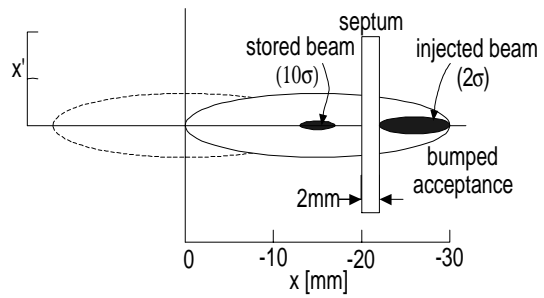

(d)

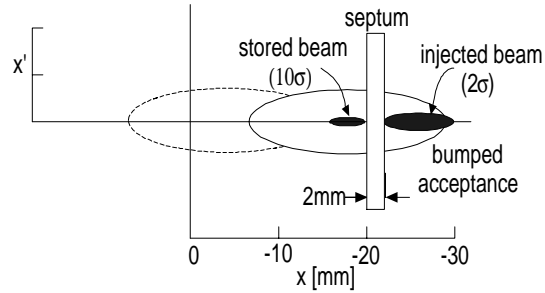

FIG. 7. Injection process: (a) before injection with a $15 \mathrm{~mm}$ acceptance, (b) during injection with a $15 \mathrm{~mm}$ acceptance, (c) before injection with a $10 \mathrm{~mm}$ acceptance and a $4 \mathrm{~mm}$ closed orbit offset, and (d) during injection with a $10 \mathrm{~mm}$ acceptance and a $4 \mathrm{~mm}$ closed orbit offset.

narrow region in parameter space where we could get a reasonable injection efficiency.

A comparison of the relative injection efficiency is made for the ring before and after the optic was corrected. The results can be seen in Fig. 8. Without changing the injector, the injection rate was measured as a function of the relative distance between the stored beam and the injected beam. This was done by incrementally shifting the stored beam further toward the injected beam using a closed orbit bump. At each bump amplitude the measured injection efficiency is significantly better for the lattice with corrected optic.

The reduction in the injection efficiency is not due to the correction of the $\beta$ function at the injection point, because even with the uncorrected optic the horizontal $\beta$ beating is small. We suspected that the reduction in injection efficiency was due to a reduction in the transverse dynamic aperture resulting from the periodicity breaking. Figure 9 shows a numerical simulation of the dynamic aperture for the original fitted model and the fitted model after periodicity restoration. The only errors included in the model are the fitted gradient errors. There are no coupling or higher-order multipole errors included. Particles are tracked on-energy and without synchrotron oscillations for 512 turns or until lost. The dynamic aperture is substantially larger for the lattice with the corrected optic.

The dynamic apertures plotted in Fig. 9 are larger than the storage ring acceptances drawn in Fig. 7. The

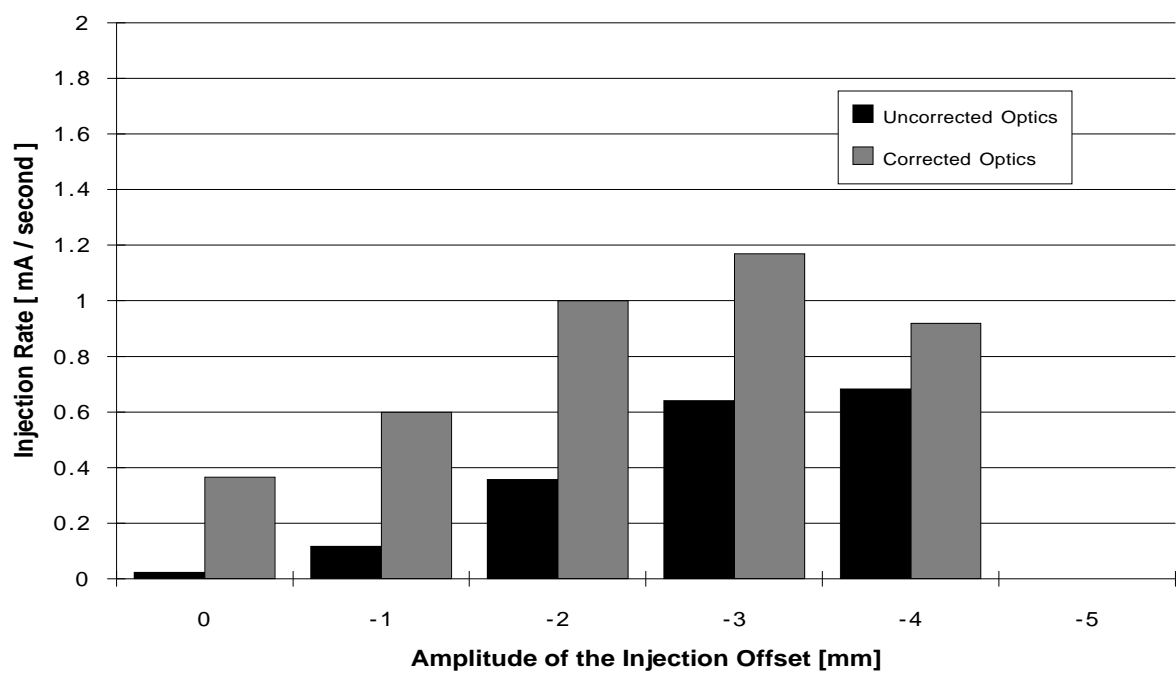

FIG. 8. A comparison of the injection rate, before and after the optic was corrected, as a function of the orbit distortion in the injection straight. (More negative means closer to the injection septum.) 


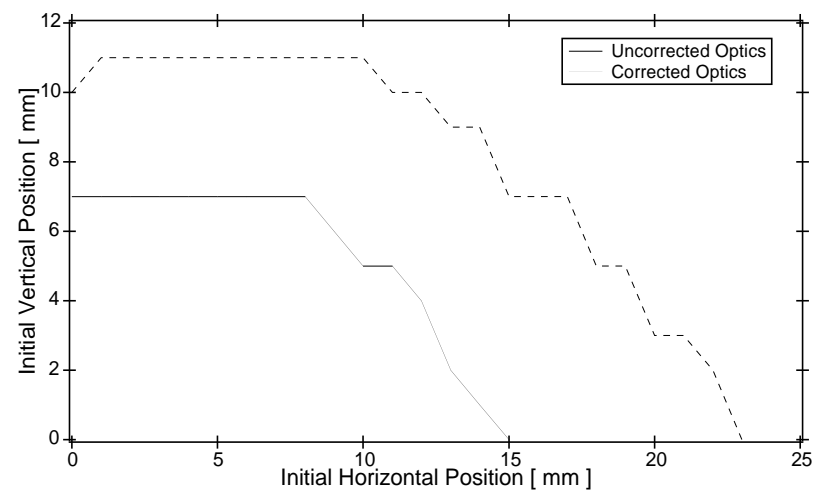

FIG. 9. A comparison of the calculated dynamic aperture before and after the optic is corrected.

calculated apertures are probably larger than the actual ones due to the fact that we included only gradient errors in the machine. So there is some uncertainty about the absolute magnitude of the aperture. Nevertheless, Fig. 9 demonstrates that there is a large reduction in the dynamic aperture for the uncorrected optic.

\section{EFFECT OF PERIODICITY BREAKING ON RESONANCE EXCITATION}

In this section we show that the measured distortion of the beam tail distribution, resulting from unallowed resonances being excited, is reduced after the optic is corrected. When excited, structural resonances may alter the behavior of particles in the beams tail. Resonances may cause particles to increase and decrease their transverse amplitudes or to be trapped at large amplitudes (for instance, particles may get trapped in resonance islands). Therefore by monitoring changes in the beam tails as the betatron tunes are varied it is possible to observe the onset of resonances.

The way in which we monitor the tails is by limiting the transverse physical aperture with a beam scraper and measuring the beam lifetime as a function of the betatron tunes. If resonances are present in the vicinity of the tunes and there is a change in the beam shape, there will be a change in the number of particles that hit the scraper when they make large amplitude excursions resulting in a change in the beam lifetime. Thus if we vary the betatron tunes while simultaneously observing the beam lifetime we will see the lifetime drop or rise when we move onto excited resonances. The experimental technique is very similar to that which was used in VEPP-4 [13] to measure the effect of the beam-beam force on the tails of the beam. A beam loss monitor consisting of two plastic scintillators with photomultiplier tube outputs in coincidence ( $\gamma$ telescope) was located just downstream of a horizontal and vertical scraper [14]. The $\gamma$ telescope detects gamma radiation emitted when electrons hit the scraper. The count rate detected is proportional to the rate at which particles hit the scraper and is linearly related to beam lifetime. Therefore by observing the change in the ratio of the beam current to the detector count rate as a function of betatron tune we can observe a change in lifetime and thus the onset of resonance excitation.

Our experimental procedure was the following. We would first change the tunes by changing two families of quadrupoles, $Q F$ and $Q D$, according to a previously measured transfer matrix. After the quadrupole fields have settled we measured the beam current and the count rate in the detector for a $1 \mathrm{sec}$ interval. (The whole process is automated and takes about $2 \mathrm{sec}$ per tune point.) Then move on to the next tune. In order to check how well the predicted tunes agree with the measured tunes we would periodically measure the tunes.

There are two advantages of measuring the count rate and current verses a direct measurement of the lifetime. First, the measurement is fast. We can scan nearly 2000 tune values per hour. The second advantage is the fluctuation in the detector count rate is small (roughly 3\%) which is due to the large detector count rate $(\sim 1 \mathrm{KHz}$ for a $10 \mathrm{~h}$ lifetime with a beam current of $5 \mathrm{~mA}$ ). The combination of a fast measurement and high sensitivity makes this technique more attractive than measuring the beam lifetime directly.

\section{A. Before periodicity was restored}

We chose to scan in a region of tune space where two resonances are present: $5 \nu_{x}=72$ (allowed by periodicity) and $3 \nu_{x}=43$ (unallowed by periodicity). The first scan was made with the lattice before the periodicity was restored. The scan covered a rectangular region in tune space $\left(14.3<\nu_{x}<14.45\right.$ and $\left.8.1<\nu_{y}<8.15\right)$. Within this region we scanned 101 horizontal tune values by six vertical tune values $\left(\Delta \nu_{x}\right.$ steps of 0.0015 by $\Delta \nu_{y}$ steps of 0.01).

Figure 10 (top row) shows the results of the scan. Three resonances can be seen in the scan:

$$
\begin{array}{ll}
5 \nu_{x}=72 & \text { allowed, } \\
3 \nu_{x}=43 & \text { unallowed, } \\
2 \nu_{x}+\nu_{y}=37 & \text { unallowed. }
\end{array}
$$

In particular, it is clear from Fig. 10 (top row) that resonance $3 \nu_{x}=43$ is largely excited.

\section{B. After periodicity was restored}

The second scan was made after we restored the periodicity and the results can be seen in Fig. 10 (bottom row). Looking at the figure it is clear that the resonance $3 \nu_{x}=43$ has been greatly suppressed down below the level of the allowed resonance, $5 \nu_{x}=72$. One can also see from the figure that the coupling resonance $2 \nu_{x}+$ $\nu_{y}=37$ seems to be of the same amplitude as before. This is not surprising considering that this is a coupling resonance and when the periodicity was restored we made 

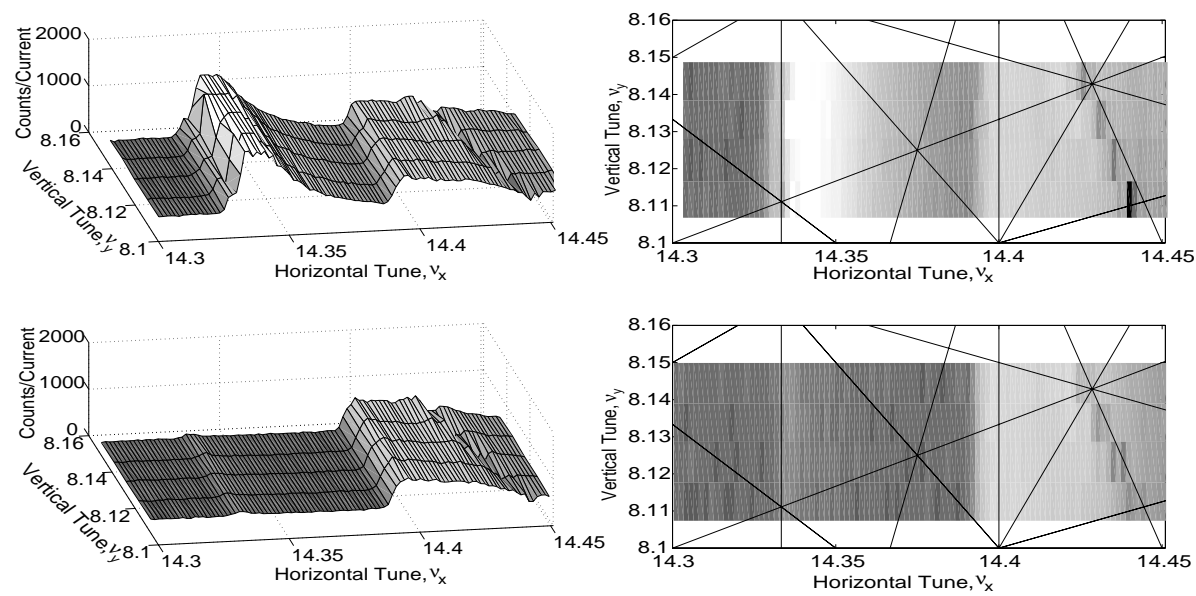

FIG. 10. Tune scans before (top) and after (bottom) the optic is corrected. At each horizontal and vertical tune, the count rate (as measured in a gamma counter) divided by beam current is plotted. The two data sets are drawn in both a hill (left) and a contour (right) plot. All resonances up to 5th order are drawn on the contour plot.

no attempt to correct the coupling in the machine. In Fig. 11 a two dimensional plot is shown where the vertical axis is count rate divided by current and the bottom axis is horizontal tune. The vertical tune is kept roughly constant at $\nu_{y}=8.15$. In this picture we can more clearly see the relative amplitudes of the various resonances. A dramatic reduction in the $3 \nu_{x}$ resonance can be seen after periodicity is restored.

We also made a larger scan of the tune region $(14.1<$ $\nu_{x}<14.45$ and $\left.8.1<\nu_{y}<8.4\right)$. We found that in the case of the lattice with periodicity restored we are easily able to move over this large region of tune space while observing very few resonances being excited. The results of the tune scan are displayed in Fig. 12. Besides the resonances mentioned one also sees the coupling resonance $\left(\nu_{x}=\nu_{y}\right)$. It is remarkable how clean the picture looks and how easily one can move over the tune space when one has a periodicitic machine.

This is not the case with the lattice before the periodicity is broken. We found that the beam was not stable in

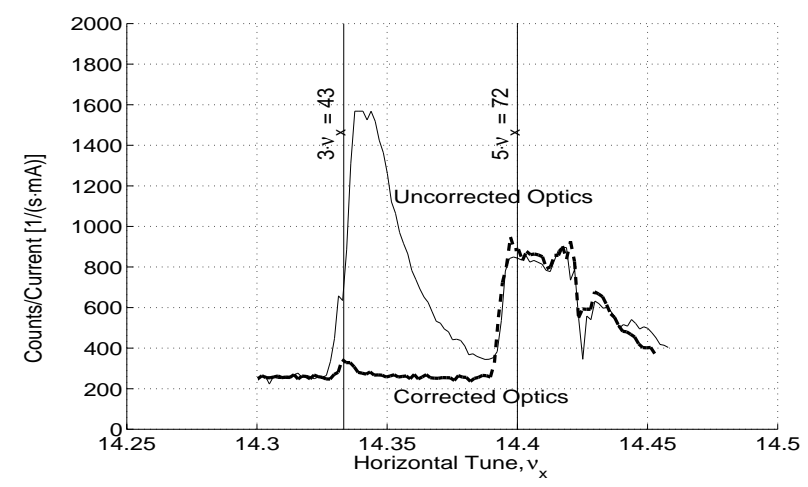

FIG. 11. Horizontal tune scans before (top) and after (bottom) the optic is corrected. The vertical tune is kept constant at 8.15. At each horizontal and vertical tune, the count rate (as measured in a gamma counter) divided by beam current is plotted. many areas of the scanned tune space. In fact, we found that there were a number of areas in tune space that could not be scanned without loosing the beam.

\section{Interpretation of the tune scan data}

The effects of periodicity breaking on the behavior of the tails at different tunes are illustrated with the help of phase space plots of the fitted lattices. Figure 13 shows the horizontal phase spaces next to the $3 \nu_{x}=43$ and $5 \nu_{x}=72$ resonances. The left side shows the phase space of the optic before periodicity was restored, the right side shows the phase space of the ideal optic.

Let us first consider the $5 \nu_{x}$ resonance (lower figures): The resonance islands are even visible in the unperturbed case, as expected from the resonance condition of the periodicitic machine. These islands remain unchanged when breaking the periodicity. Only the outer part of the phase space is distorted by the $8 \nu_{x}=115$ resonance. In the vicinity of the $3 \nu_{x}$ resonance the situation is completely different. Whereas in the unperturbed case there is no distortion at all, the inner phase space becomes completely distorted by the islands of the third integer resonance when the periodicity is broken.

We expect that with islands in the phase space, particles can get captured in the tails either through gas scattering or intrabeam scattering. This distorts the distribution of the beam, leading to an increase in the beam loss rate. From the phase space pictures in Fig. 13 we expect that the tail distributions and the beam loss rate in the vicinity of the $5 \nu_{x}$ resonance would remain roughly the same before and after periodicity was restored. While in the vicinity of the $3 \nu_{x}$ resonance the tail distributions and count rate would be higher before the periodicity was restored. This is consistent with what we observed experimentally (see Fig. 11). A close observation of Fig. 11 reveals that even after the periodicity was restoredthere is still a 

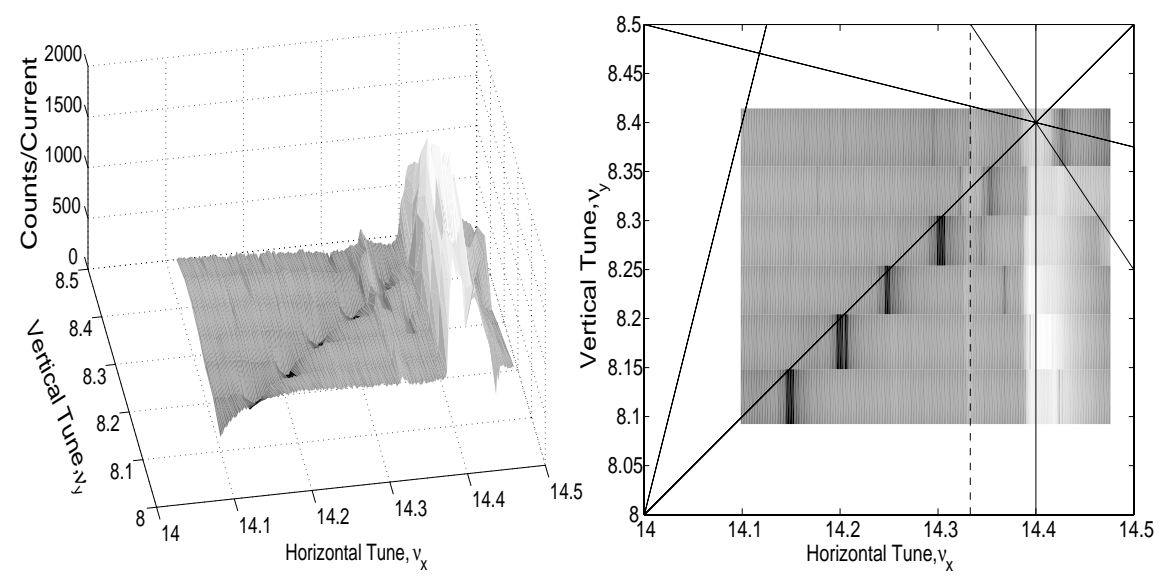

FIG. 12. Larger area tune scans of the lattice after the optic is corrected. At each horizontal and vertical tune, the count rate (as measured in a gamma counter) divided by beam current is plotted. Data are drawn as a hill plot (left) and as a contour plot (right). All "allowed" resonances up to 5th order are plotted (solid line). The resonance $\nu_{x}=43$ is plotted as a dashed line.

small increase in count rate near the $3 \nu_{x}$ resonance. This indicates that periodicity is not perfectly restored.

The decrease in count rate near the coupling resonance (see Fig. 12) can be explained in the following way: When the tunes cross the coupling resonance, the core of the beam changes size (increases vertically and decreases horizontally) with an overall reduction in the core density. If intrabeam scattering is responsible for populating the tails the reduction in the core density will result in a decrease in tail population and thus a lower count rate.
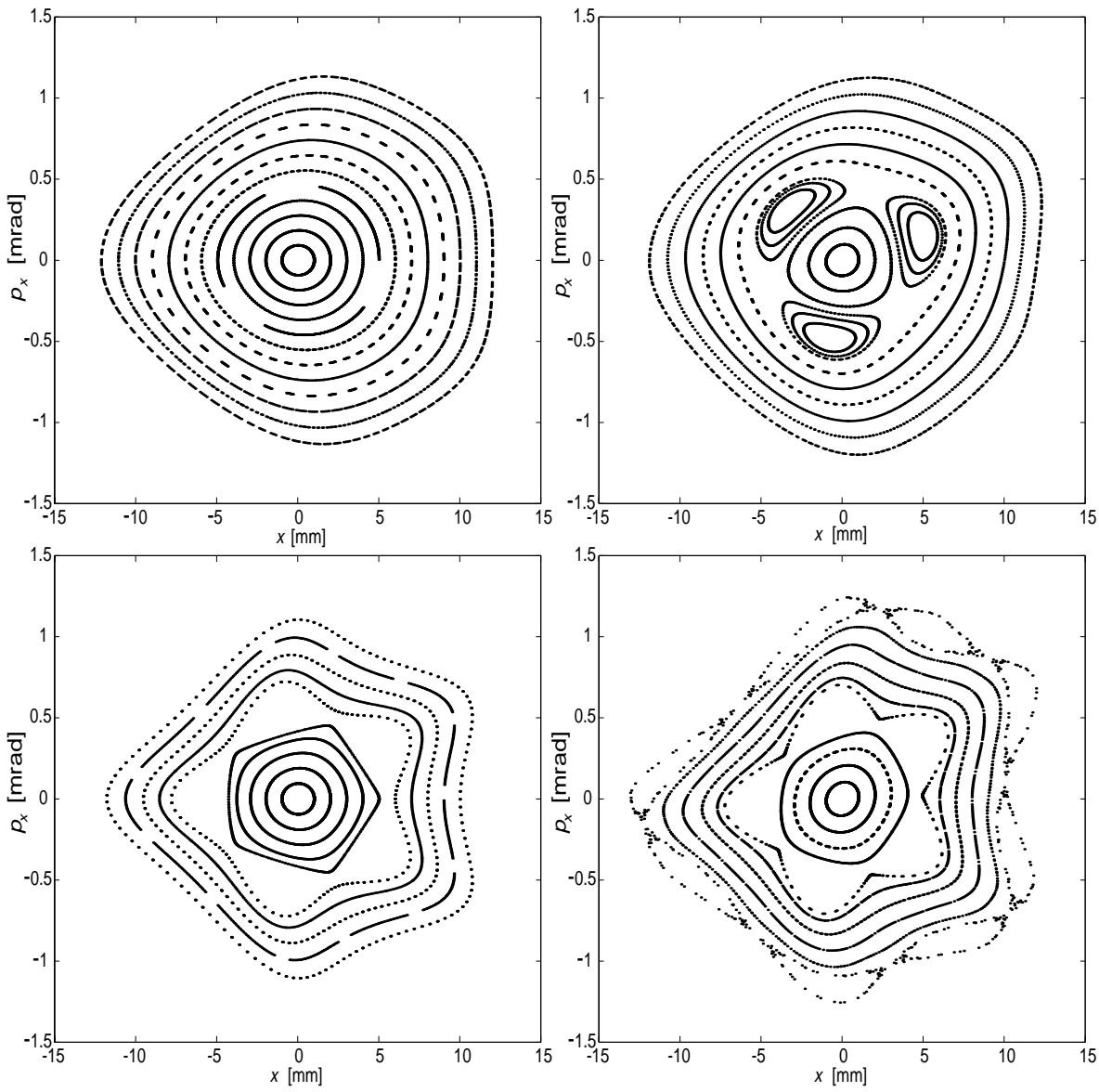

FIG. 13. Comparison of the horizontal phase space for an ideal machine (left) and one with uncorrected optic (right). The plots are made with the model tuned in the vicinity of the $3 \nu_{x}=43$ (upper) and the $5 \nu_{x}=72$ (lower) resonances. 

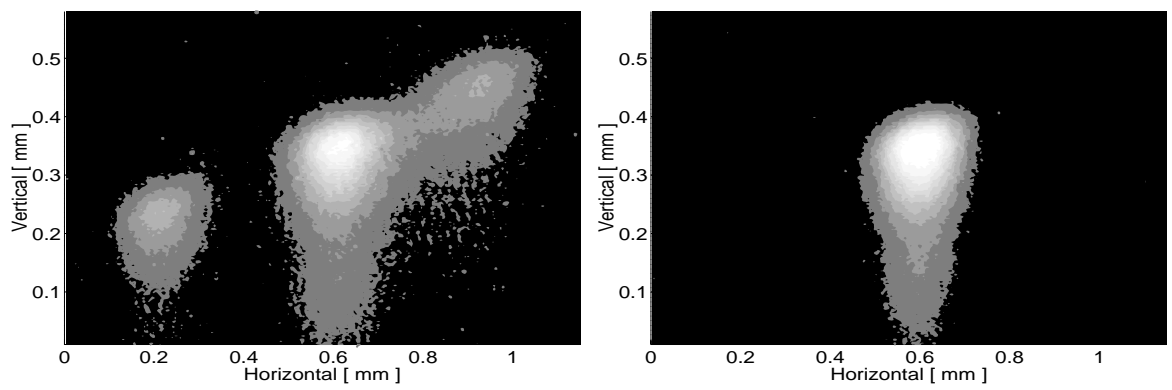

FIG. 14. Synchrotron radiation image of the beam near the $3 \nu_{x}$ resonance. Left is the situation before the optic is corrected and right is the situation after the optic is corrected. (The plane of the camera is rotated with respect to the plane of the beam.) Also there is a distortion in the light optic in the vertical plane that is responsible for the image's vertical asymmetry.

\section{Beam profile monitor}

We have the ability to observe the beam distribution with a beam profile monitor. This monitor images the synchrotron radiation that is emitted from a bending magnet and represents roughly a one-to-one image of the beam. An image of the beam near the $3 \nu_{x}$ resonance can be seen in Fig. 14 before (left) and after (right) the periodicity was restored. What we see is that in the lattice before the periodicity was restored the beam "splits" into several spots. However, that was not the case after the periodicity was restored.

\section{E. Interpretation of the beam profile measurement}

Again a phase space plot of the fitted lattice is used to help us understand the image. In Fig. 15 we have plotted the horizontal phase space of the lattice without the periodicity restored. The tracking point of the phase space corresponds to the observation point of the beam. As we expect there are three islands in the phase space. One can imagine that if the islands are populated the projection onto the normal space would look something like the image. The reason that one sees only three spots and not four is that when one projects the phase space onto the $x$ axis two islands overlap. The phase space plot is only meant to serve as a qualitative understanding of the image. We make no quantitative statements about the distribution.

\section{CONCLUSION}

The periodicity of the linear lattice is clearly important for the ALS. The actual linear lattice is accurately determined by analyzing orbit response matrices. From this analysis we have found power supply errors and orbit offsets in sextupoles which lead to a beating of the vertical $\beta$ function of $19 \%$. Knowing the errors in the machine we then adjusted quadrupoles in the machine to restore the lattice periodicity. After the quadrupoles were adjusted, beating of the $\beta$ function was reduced to less than $1 \%$.

We find that by restoring the lattice periodicity there have been several beneficial effects on the machine performance. First, the excitation of unallowed nonlinear resonances is suppressed. Second, the injection efficiency is improved. As a result, we are able to tune the machine anywhere (except near integer and half-integer resonances) with stable beam. Finally, we find that by restoring the lattice periodicity the beam profile remains nearly constant near unallowed resonances.
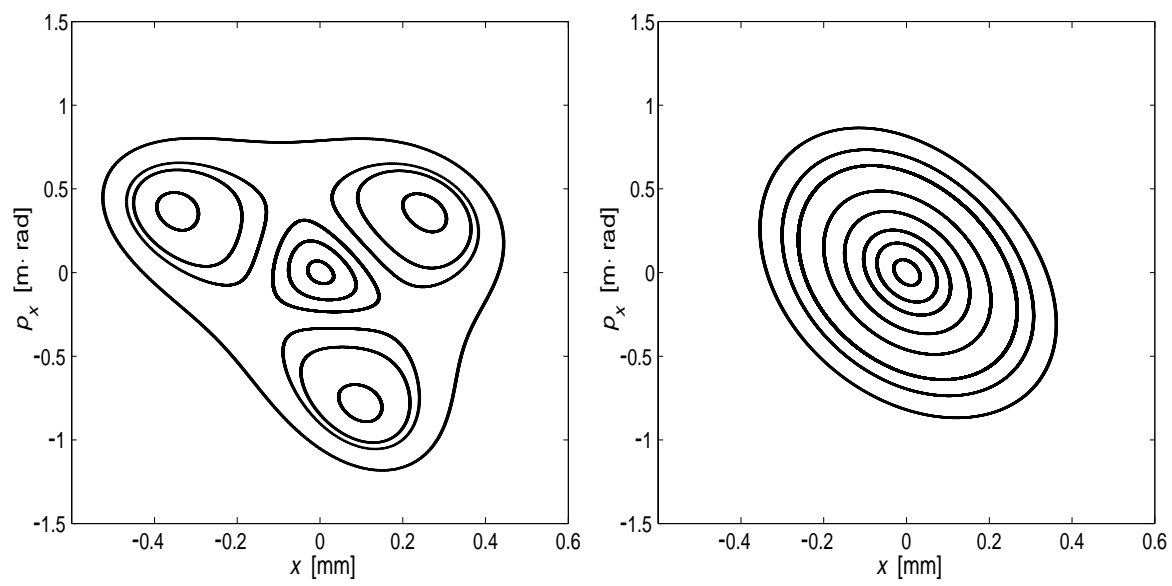

FIG. 15. Horizontal phase space of the lattice plotted at the observation point. Lattices are tuned to $\nu_{x}=14.3338$ and $\nu_{y}=8.15$. The left plot shows the phase space with the uncorrected optic. The right plot shows the phase space for the ideal machine. 


\section{ACKNOWLEDGMENTS}

The authors would like to thank the staff at the ALS, particularly Alan Jackson for his encouragement and support of the work and with whom we had many interesting discussions. Also, we would like to thank Alan for making high precision power supply measurements. We wish to thank Gary Krebs for setting up the radiation monitors used in the tune scan experiments. We wish to thank Greg Portmann for helping to automate the tune scan measurements. Also we wish to thank Greg, Jim Hinkson, and Jim Johnson for reducing the noise levels of the BPMs. We would like to thank Sasha Zholents for suggesting the tune scan technique for measuring resonance excitation. We wish to thank Sam Krinsky at BNL for encouraging one of the authors (J.S.) to participate in this work. We would also like to thank Martin Lee and Jeff Corbett at SLAC for interesting discussions. This work was supported by the Director, Office of Energy Research, Office of Basic Energy Sciences, Materials Sciences Division of the U.S. Department of Energy, under Contract No. DE-AC0376SF00098.

[1] Lawrence Berkeley National Laboratory Report No. LBNL/PUB-5172, 1986 (unpublished).

[2] W. J. Corbett, M. Lee, and V. Ziemann, in Proceedings of the 1993 Particle Accelerator Conference, Washington, DC (AIP, Woodbury, NY, 1993), p. 108.

[3] J. Safranek, in Proceedings of the 1995 Particle Accelerator Conference, Dallas, TX (IEEE, Piscataway, NJ, 1996), p. 2817.

[4] J. Safranek, Nucl. Instrum. Methods Phys. Res., Sect. A 388, 27 (1997).

[5] J. Bengtsson and M. Meddahi, in Proceedings of the 1994 Particle Accelerator Conference, London (World Scientific, Singapore, 1994), p. 1021.

[6] D. Robin, J. Safranek, G. Portmann, and H. Nishimura, Proceedings of the 1996 Particle Accelerator Conference, Barcelona (IOP, Bristol, 1996), p. 971.

[7] M. P. Woodley et al., Stanford Linear Accelerator Conference Report No. SLACPUB 3086, 1983 (unpublished).

[8] Alan Jackson (private communication).

[9] E. Ciapala et al., IEEE Trans. Nucl. Sci. 26, 3571 (1979).

[10] Advanced Light Source Parameter List, 1992 (unpublished), VIIIth ed.

[11] Measurements made by Alan Jackson.

[12] J. Bengtsson and E. Forest, in Advanced Beam Dynamics Workshop on Effects of Errors in Accelerators, Their Diagnosis and Corrections, edited by A.W. Chao, AIP Conference Proceedings No. 255 (AIP, New York, 1992), p. 229.

[13] A. B. Temnykh, in Proceedings of the IXth ALL-Union Meeting on Accelerators of Charged Particles, Dubna, 1984, Vol. 2, p. 163 (INP Report No. INP 84-131, 1984).

[14] D. Robin et al., in Proceedings of the 1995 Particle Accelerator Conference, Dallas, TX (Ref. [3]), p. 2789. 